\title{
ANALYSIS AND MEASUREMENTS OF EMITTANCE DILUTION FROM VACUUM WINDOWS IN THE FERMILAB RECYCLER TRANSFER LINES*
}

\author{
C. Johnstone ${ }^{\#}$, C. Gattuso, D. Johnson, M. Syphers, FNAL, Batavia, IL 60510, USA \\ K. Paul, UIUC, Urbana, IL 61801, USA
}

\begin{abstract}
Round-trip beam transfers between the Fermilab Main Injector and Recycler (antiproton accumulator) have been plagued by an emittance dilution of about a factor of 2 with corresponding beam loss. The source of the large dilution was traced to two $\mathrm{Ti}$ vacuum windows, one installed in each of the two transfer lines that connect the machines for beam injection/extraction. The dilution can be accounted for by calculating the increase in beam divergence due to multiple scattering in the windows followed by an amplitude mismatch that serves to further enhance the instantaneous dilution immediately downstream of the window. This work presents the analytical basis for the phase-space, or emittance, dilution due to the window, the subsequent optics mismatch, and then presents an analysis of beamline measurements that evidences the effect and validates the analytical argument. The data showed a strong skew quadrupole component in the transfer line optics that initially complicated the analysis, but was effectively eliminated in some of the data samples acquired under specific beam conditions.
\end{abstract}

\section{INTRODUCTION}

For some time beam transfers of $8-\mathrm{GeV}$ protons between the Main Injector synchrotron and the Recycler permanent-magnet storage ring were plagued by beam loss and immediate emittance dilution, with evidence of an amplitude function mismatch between the accelerators presumably generated in the transfer lines. Of concern were Titanium (originally beryllium) vacuum windows, estimated to be $\sim 3$ mil thick, installed in each transfer line. The effects on emittance and subsequent secondmoment oscillation amplitudes due to beam passing through these windows will be first derived followed by experimental verification in a later section.

The emittance dilution can be derived in several forms. All forms depend on solving the linear equations of motion (linear magnetic fields: quadrupole and dipoles fields) piecewise which results in constraining the coordinates of particle motion to an elliptical envelope given by:

$$
\varepsilon=\gamma x^{2}+2 \alpha x x^{\prime}+\beta x^{\prime 2}
$$

where $\beta, \alpha$, and $\gamma$ (which is $\left(1+\alpha^{2}\right) / \beta$ ) are the so-called Courant-Snyder parameters and $\varepsilon$ is the area of the ellipse. These lattice parameters evolve according to the optical solutions along the line, but the area of the ellipse, or total phase space, remains conserved, and in a predominately linear system, particles remain on their respective elliptical contour. Further, if the system is periodic, the phase ellipse is also repetitive, and particles move around the given contour according to the phase advance of the lattice.

\section{EMITTANCE DILUTION FROM MULTIPLE SCATTERING}

When beam passes through material, the dominant process is Coulomb multiple scattering, which changes the direction but not the energy or position of the incident beam particle if the material is thin. Multiple scattering is a statistical process which is azimuthally symmetric about the particle's trajectory (this symmetry will be exploited later). The phase space of a distribution immediately after a thin window is expanded in one coordinate only, $x$ '.

The change in a particle's phase coordinates is represented by a discrete jump to a different ellipse generating, on average, a clearly different aspect ratio; the change in the distribution is governed by the statistics of the scattering process. Since the original distribution is an even function in $\mathrm{x}$ and $\mathrm{x}^{\prime}$, and the scattering process is an even-function convolution of the original distribution, the rms of the distribution is a particularly powerful description - there is not only an rms value for $\mathrm{x}$ and $\mathrm{x}$ ', but also an rms emittance, $\varepsilon$, and an rms multiple scattering angle specific to the composition and thickness of the window (values for multiple scattering angles will be given later in this paper).

\section{Statistical Ensemble Description}

This derivation is facilitated by conversion to "normalized" coordinates which always reduces the ellipse to a circle; the phase space of the beam just upstream of the vacuum window is circular when expressed in $\left\{x,\left(\beta x^{\prime}+\alpha x\right)\right\}$ coordinates. (One plane conveniently remains the original transverse coordinate.) Initially we assume an otherwise perfectly matched beam between the two accelerators, and look at the effect of the particles passing through a vacuum window. The process is depicted in Figure 1.

\footnotetext{
**Work supported by the Universities Research Association, Inc., under contract DE-AC02-76CH00300 with the U.S. Department of Energy.

\#cjj@fnal.gov
} 


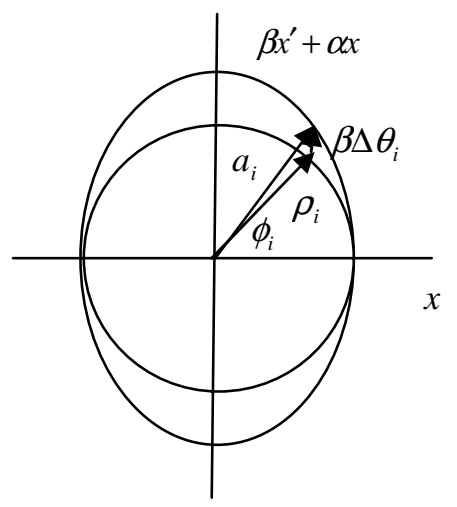

Figure 1. Phase space in normalized coordinates showing the new oscillation amplitude $a_{\mathrm{i}}$ a single particle may acquire after multiple scattering in a window.

The particles are initially following trajectories around the inner circle, each has a radius $\rho_{\mathrm{i}}$, with a corresponding transverse amplitude of :

$$
\sigma_{0}(x)=\left\langle x^{2}\right\rangle^{1 / 2}=\left\langle\rho^{2} / 2\right\rangle^{1 / 2} .
$$

Upon passing through the window, the ith particle's amplitude will change from $\rho_{\mathrm{i}}$ to $\mathrm{a}_{\mathrm{i}}$ according to:

$$
a_{i}^{2}=\rho_{i}^{2}+\left(\beta \Delta \theta_{i}\right)^{2}-2 \beta \rho_{i} \Delta \theta_{i} \cos \phi_{i}
$$

where $\beta$ is the amplitude function at the window, and $\Delta \theta_{i}$ is the final scattering angle through the material due to multiple Coulomb interactions. The phase angle, $\phi_{\mathrm{i}}$, depends upon the phase of the particle in its phase space oscillation, and is randomly distributed among all the particles, and is thus uncorrelated with either $\rho$ or scattering angle. Therefore, after averaging over all the particles in the beam, we have

$$
\begin{aligned}
\left\langle a^{2}\right\rangle & =\left\langle\rho^{2}\right\rangle+\beta^{2}\left\langle\Delta \theta^{2}\right\rangle-2 \beta\langle\rho \Delta \theta \cos \phi\rangle \\
& =\left\langle\rho^{2}\right\rangle+\beta^{2}\left\langle\Delta \theta^{2}\right\rangle-2 \beta\langle\rho\rangle\langle\Delta \theta\rangle\langle\cos \phi\rangle \\
& =\left\langle\rho^{2}\right\rangle+\beta^{2}\left\langle\Delta \theta^{2}\right\rangle
\end{aligned}
$$

Note that the scattering angle, $\Delta \theta$, and $\cos \phi_{i}$ independently average to zero, as there is no preferred direction. The resulting phase space distribution subsequently tumbles through the lattice of the beamline and through the downstream machine, resembling an amplitude function mismatch in that a transverse beam profile monitor will see a "quadrupole oscillation" ensue at twice the betatron tune until the coherent oscillation amplitude dies away due to filamentation. Filamentation, as depicted in Figure 2, proceeds through unavoidable field nonlinearities, which simply implies differences exist in phase advance as a function of particle amplitude. (Chromatic correction sextupoles are usually the largest contributor to this effect.)
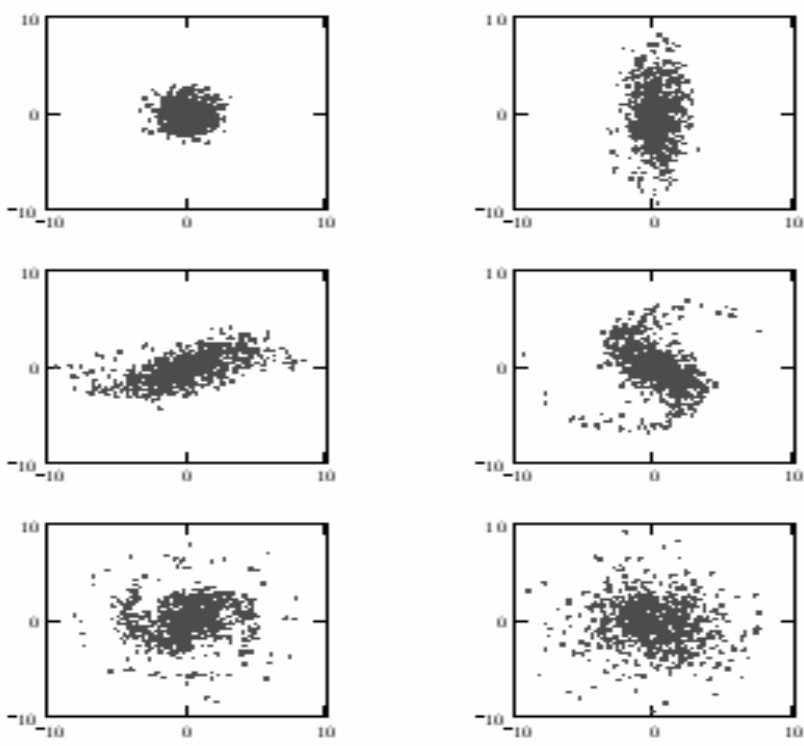

Figure 2. From top left to bottom right: phase space distributions, a) before undergoing scattering in the window, c)-e) during filamentation, and f) after filamentation is complete. For each plot the horizontal axis is $\mathrm{x}$ and the vertical axis is $\left(\beta \mathrm{x}^{\prime}+\alpha \mathrm{x}\right)$. The scales are arbitrary units.

Each particle now oscillates in phase space with its new betatron amplitude (or single particle invariant ellipse) acquired in the scattering process. If the new phase space distribution has not been "re-matched" after the window, then, through filamentation, a final equilibrium distribution is reached within a larger matched ellipse concentric to the original one (or circle in normalized coordinates). In normalized coordinates, the new amplitude $\left\langle\alpha^{2}\right\rangle^{1 / 2}$ describes the final redistribution. Again, since the new envelope is circular,

$$
\begin{aligned}
& \sigma_{f}^{2}(x)=\left\langle x^{2}\right\rangle_{\text {final }}=\left\langle\rho^{2} / 2\right\rangle+\frac{1}{2} \beta^{2}\left\langle\Delta \theta^{2}\right\rangle . \\
& \text { or } \\
& \sigma_{f}^{2}(x)=\sigma_{o}^{2}(x)+\frac{1}{2} \beta^{2} \theta_{r m s}^{2} .
\end{aligned}
$$

In the second equation, $\theta_{r m s}$ is the rms scattering angle due to multiple Coulomb interactions in the window material as projected into a transverse plane (the specific equation used to compute this angle is given later in the text.) The rms emittance enhancement then follows from:

$\Delta \varepsilon_{r m s}=\frac{\Delta \sigma^{2}}{\beta} \pi=\frac{1}{2} \beta \theta_{r m s}^{2}$,

or

$\Delta \varepsilon_{N}=3 \beta \theta_{r m s}^{2}(\beta \gamma) \pi$

for the 95\% normalized emittance (this derivation can also be found in references [1]). One observation which 
stands out is that the increase in emittance is not dependent on the incoming emittance upstream of the window, only on the $\beta$ function, or optics, at the vacuum window itself. This is an interesting result and derives from the fact that the scattering angle adds in quadrature to the beam divergence--thus the higher the beam divergence, or the smaller the beam size as indicated by the beta function, the lower the increase in emittance.

\section{Coulomb Multiple Scattering}

The projected rms scattering angle resulting from multiple Coulomb interactions in a material can be estimated using[2]

$$
\theta_{r m s}=\frac{13.6 \mathrm{MeV}}{p \beta} \sqrt{\frac{l}{L_{r a d}}}\left[1+0.038 \ln \left(\frac{l}{L_{r a d}}\right)\right]
$$

where $l$ is the thickness of the material, $L_{\text {rad }}$ is the radiation length (which is $3.56 \mathrm{~cm}$ for $\mathrm{Ti}$ ), $p$ the momentum of the particle, and $\beta$ here is the relativistic velocity.

With 3 mil Ti windows the predicted emittance blowup at $8 \mathrm{GeV}$ is $1.1 \pi$ and $4.2 \pi \mathrm{mm}-\mathrm{mr}$ horizontally and vertically, due to the $13 \mathrm{~m}$ and $50 \mathrm{~m}$ beta functions, respectively, at the location of the windows.

\section{EXPERIMENTAL MEASUREMENTS}

In the following data, the beam emittance has been measured using flying wires in the Fermilab Main Injector before and after completing a round-trip transfer to and from the Fermilab Recycler. The beam passes through two Ti windows in the process. The measured blowup was $\Delta \varepsilon=7.6 \pi$ (horz) $+7 \pi$ (vert) $=14.6 \pi \mathrm{mm}$-mr total-(blowup in both planes $\sim 60 \%$ ). No asymmetry was observed in the blowup. If the vertical and horizontal planes were completely mixed by the time of the measurement, then the combined expected blowup of at least $10.6 \pi \mathrm{mm}-\mathrm{mr}$ is, in fact, completely consistent with the observed dilution (about $3-4 \pi \mathrm{mm}-\mathrm{mr}$ can be attributed to nonlinearities in the accelerator systems). The mixing of the two planes was, in fact, verified through a pencil-beam measurement which evidenced blowup in the vertical plane only. Strong skew quadrupole components in the transfer line have been identified.

In a shutdown last winter the vacuum windows were completely removed and the flying wire data retaken. The large dilution disappeared leaving only the expected $3-4 \pi \mathrm{mm}-\mathrm{mr}$.

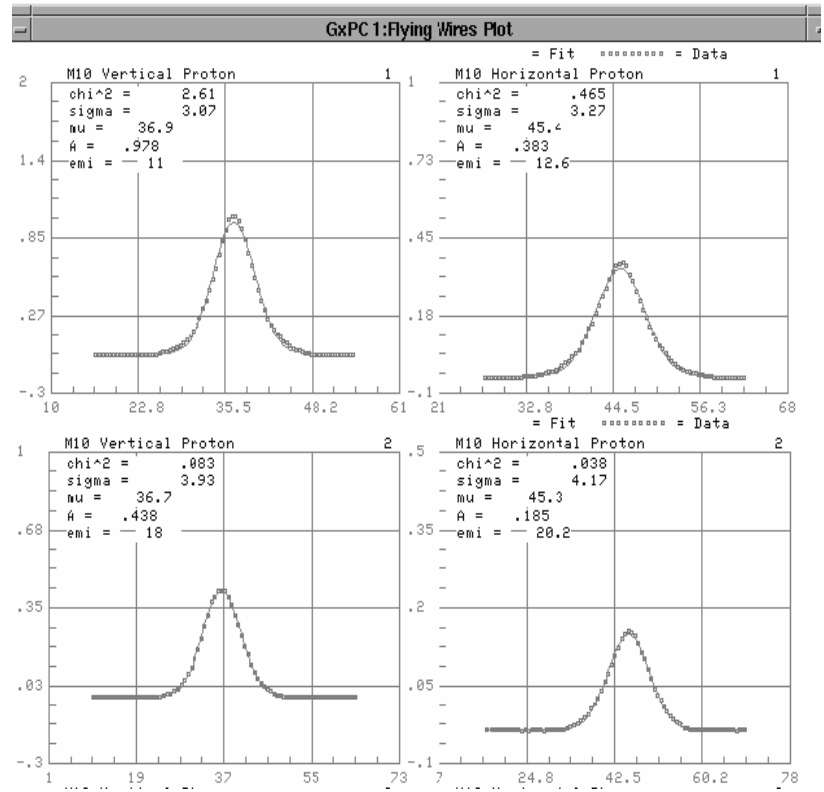

Figure 3. Flying wire data taken before and after transfer to the Recycler. The last number is the $95 \%$ normalized emittance as calculated from the data and the optics.

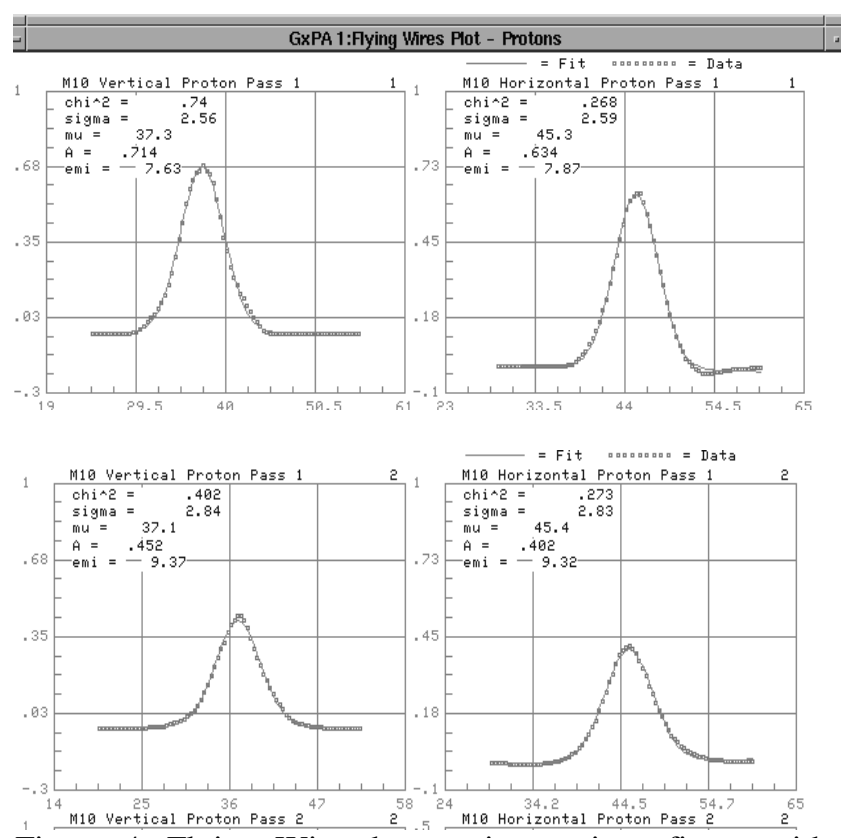

Figure 4. Flying Wire data as in previous figure with vaccum window removed.

\section{REFERENCES}

[1] D. A. Edwards and M. J. Syphers, An Introduction to the Physics of High Energy Accelerators, J. Wiley, \& Sons, Inc., New York (1993).

P. J. Bryant, Introduction to Transfer Lines and Circular Machines, CERN-84-04.

[2] Phys. Rev. D, Vol. 66, Review of Particle Physics, July 2002, 1998. 